\title{
Filtró la anastomosis esofágica. ¿Y ahora qué?
}

\author{
The Esophageal anastomosis leaked, now what?
}

Con el advenimiento de las cirugías digestivas mínimamente invasivas en las últimas décadas, demostrando su efectividad técnica, oncológica y estética, ha aumentado la necesidad por parte de especialistas y pacientes que la resolución de sus complicaciones, sean de la misma forma.

En este contexto, la filtración de anastomosis del tracto digestivo superior y particularmente de la esofagectomía, ha sido un foco de interés por su frecuencia, morbilidad y mortalidad de hasta 60,30 y $15 \%$ respectivamente ${ }^{1}$.

Durante los últimos años la instalación de prótesis metálicas auto-expansibles (SEMS) ha sido reconocida como el "Gold Standard" mínimamente invasivo para afrontar este tipo de complicaciones con un éxito del $88 \%{ }^{2-3}$, sin embargo, frente a los costos asociados a ellas y las complicaciones del procedimiento y postprocedimiento, aparece el "Endoscopic Vacuum-Assisted Closure" (EVAC), adaptado de su símil en recto descrito el $2006^{4}$ como una alternativa terapéutica.

En relación con la técnica del EVAC: deber ser realizada por un endoscopista experto, con apoyo de imágenes y sedación profunda. Una vez analizado imágenes y medido el defecto por visión endoscópica, se determina el tamaño de la esponja de poliuretano (se recomienda considerar 4-5 y 1-2 cm más de largo $\mathrm{y}$ ancho respectivamente). A través de una fosa nasal, se inserta una sonda Naso-gástrica 16 French la que se recupera por vía oral. Se sutura la esponja al extremo distal con material reabsorbible 3.0 y con una pinza endoscópica se guía hasta posicionarla intra-cavitaria o endo-luminal (idealmente en defectos múltiples o muy pequeños) fijándola a nivel nasal e iniciando la terapia a presión negativa de entre $-100 \mathrm{mmHg}$ a $-125 \mathrm{mmHg}$. Este procedimiento debe repetirse cada 72-96 h, lo que permite limpiar y disminuir la cavidad mientras se deriva el tránsito intestinal con una sonda Naso-yeyunal, asociado al tratamiento médico necesario y los controles imagenológicos protocolizados por cada unidad (Figura 1).

Algunos reportes de $2016^{1}, 2019^{5}, 2020^{6}$, metaanálisis de $2018^{7}$ y revisión sistemática de $2019^{8}$ muestran no solo lo reproducible de la técnica, sino que además, que en una estadía hospitalaria similar o menor, esta técnica ha demostrado disminución y cierre de la cavidad con menor morbilidad (OR 0,38; $\mathrm{p}=0,01)^{7}$ y mortalidad (OR 0,$\left.33 ; \mathrm{p}=0,002\right)^{7-8}$ en comparación a la utilización de SEMS, lo que apoya

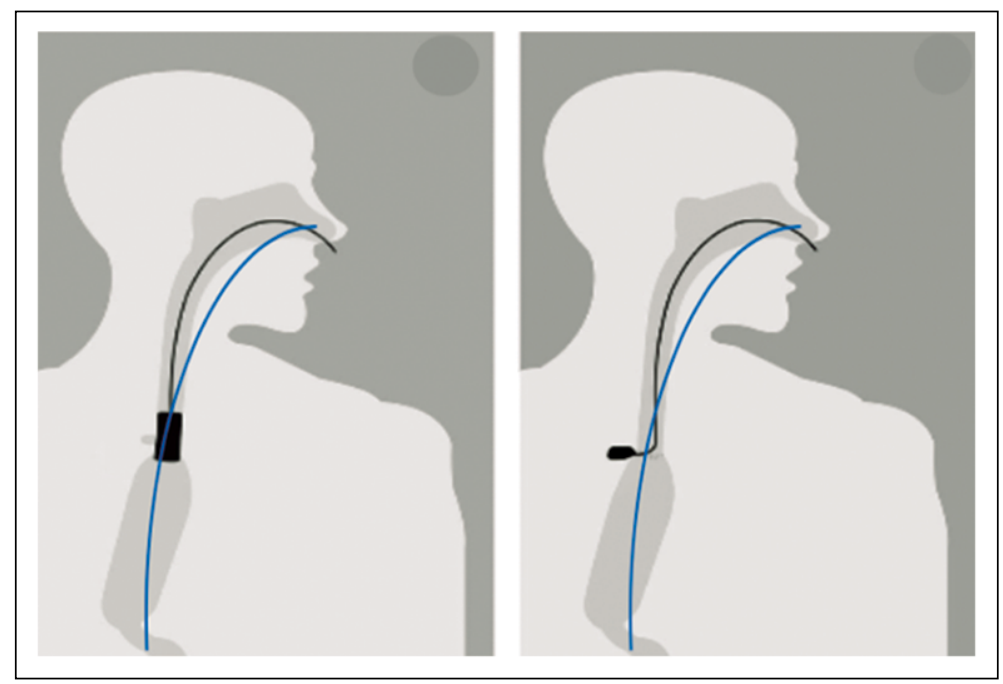

Figura 1. Esquema de instalación endoluminal e intracavitaria, más sonda Naso-yeyunal de alimentación.

la hipótesis de que, es una técnica útil y que llegó para quedarse.

Es importante considerar que a pesar de que los datos mostrados por los meta-análisis ${ }^{7}$ y revisiones sistemáticas $^{8}$ son de un grado de evidencia altos y valiosos para el uso clínico, los estudios incorporados en los mismos, son heterogéneos y con baja calidad metodológica. Lo que el estudio TENTACLE espera cambiar?.

Se realizó una búsqueda bibliográfica utilizando los siguientes términos MESH «Negative-Pressure Wound Therapy", "Esophageal Neoplasms" y "Anastomotic Leak", además de artículos similares sugeridos por PUBMED, sin encontrar referencias relacionadas de origen latinoamericano, lo que significa una gran oportunidad para aportar al conocimiento científico, con experiencias locales y estudios comparativos de mayor calidad metodológica y así ofrecer el state of the art, a nuestros pacientes.

Felipe Sandoval O. ${ }^{1}$, María Patricia Cornejo A. ${ }^{1}$, Rodney Stock L. ${ }^{1}$ y Roque Sáenz $F^{1}$

${ }^{1}$ Facultad de Medicina Universidad del Desarrollo/ Clínica Alemana, Chile. Instituto Nacional del Cáncer, Chile. 


\section{Cartas al Editor}

\section{Referencias}

1.- Mennigen R, Harting C, Lindner K, Vowinkel T, Rijcken E, Palmes D, et al. Comparison of Endoscopic Vacuum Therapy versus Stent for Anastomotic Leak After Esophagectomy. J Gastrointest Surg. 2015;19:1229-35.

2.- Van Boeckel PG, Sijbring A, Vleggaar FP, Siersema PD. Systematic review: temporary stent placement for benign rupture or anastomotic leak of the oesophagus. Aliment Pharmacol Ther 2011; 33: 1292-301.

3.- Dasari BV, Neely D, Kennedy A, Spence G, Rice P, Mackle E, Epanomeritakis E. The role of esophageal stents in the management of esophageal anastomotic leaks and benign esophageal perforations.

Ann Surg 2014; 259: 852-60.

4.- Weidenhagen R, Gruetzner ku, Wiecken T, Spelsberg F, Jauch kW. Endoscopic vacuum-assisted closure of anastomotic leakage following anterior resection of the rectum: a new method. Surg Endosc. 2008; 22: 1818-25.

5.- Jeon JH, Jang HJ, Han JE, Park YS, Seong YW, Cho S, et al. Endoscopic Vacuum Therapy in the Management of Postoperative Leakage After Esophagectomy. World J Surg. 2020;44:179-185.

6.- Cwaliński J, Hermann J. Endoscopic vacuum assisted closure of esophagogastric anastomosis dehiscence: A case report. World J Gastrointest
Endosc 2020; 12: 42-8.

7.- Rausa E, Asti E, Aiolfi A, Bianco F, Bonitta G, Bonavina L. Comparison of endoscopic vacuum therapy versus endoscopic stenting for esophageal leaks: systematic review and meta-analysis. Dis Esophagus. 2018;31(11).

8.- Verstegen M, Bouwense S. Management of intrathoracic and cervical anastomotic leakage after esophagectomy for esophageal cancer: a systematic review. World Journal of Emergency Surgery 2019;14:17.

9.- Treatment of Anastomotic Leakage after Esophagectomy (TENTACLE study) (NCT03829098). 2019. https://clinicaltrials.gov/ct2/show/ NCT03829098. 